Methods: We used Medicare claims data for HCV patients to assess the incidence rate of ADRDwith and without exposure to periodontal disease between 2014 and 2017. Diagnosis of periodontal disease, HCV, and ADRD were based on ICD-9 and ICD-10 codes. A Cox multivariate regression model was used to estimate the association between periodontal disease and development of ADRD, controlling for age, gender, race, ZIP-level income and education, and medical comorbidities.

Results: Of the 440,578 patients in the dataset, the incidence rate of ADRD in the periodontal disease group was higher compared to those without periodontal disease $(10.77 \%$ vs. $9.27 \%, p<0.001$, and those with periodontal disease developed ADRD earlier compared to those withoutperiodontal disease (1.15 vs. 1.78 years, $p<0.001$ ). The hazard of developing ADRD was 1.23 times higher in those with periodontal disease $(95 \% \mathrm{Cl}, 1.19$ to $1.27, \mathrm{p}<0.001)$ after adjusting for all covariates, including age.

Conclusion: Periodontal disease increased the risk of developing ADRD in HCV patients in anational Medicare claims dataset.

\title{
505 - Reversible Dementia caused by Hypothyroidism - a case report
}

\author{
Diogo Almeida, Guadalupe Marinho
}

OBJECTIVES: Reversible causes are thought to explain about eight percent of all dementias. Hypothyroidism is one of the most important causes of potentially reversible dementia. Deficits in memory, psychomotor slowing, general intelligence, and visuoperceptual skills are particularly involved and may not fully recover. We review a clinical case of a reversible dementia caused by hypothyroidism, in a patientfollowed in our institution.

METHODS: Case report using clinical files, and brief literature review using Pubmed database, searching for the keywords "reversible dementia", "hypothyroidism" and"psychosis".

RESULTS: We present a case of a 76-year-old female patient admitted in our acute unitwith visual and auditive hallucinations and persecutory delusional ideation for 1 month. There was no previous psychiatric history. The patient was fully oriented in space, time and person, but there were clear memory deficits and sensitivity to antipsychotics. We used the Montreal Cognitive Assessment (MoCA) and the Frontal Assessment Battery (FAB), having the patient scored 17 on the former and 3 on the latter, failing in all tests except for grasping. On the blood tests, fT3 and fT4 levels werenear 0 and TSH was $40 \mu \mathrm{g} / \mathrm{dL}$. We then discovered that the patient had a thyroidectomy 25 years ago and had been doing replacement treatment since then buthad discontinuated treatment on the previous six months. We also did magnetic resonance imaging that showed frontal microcirculatory changes but without clear atrophy. The patient was treated with aripiprazole $30 \mathrm{mg}$ and levothyroxine $0,150 \mathrm{mg}$, being discharged after 1 month, without psychotic symptoms. About 1 year after, we repeated MoCa and FAB, scoring 27 and 16, respectively. The psychotic symptoms didn't recur even after the antipsychotic discontinuation.

CONCLUSION: We present a case of hypothyroidism induced dementia with psychotic symptoms, that fully recovered with thyroid replacement treatment, without previous neurological or psychiatric history. 